\title{
A Time-Delayed Neural Network Model for Neutron TOF Spectrum Measurements
}

\author{
M. I. Khalil
}

\begin{abstract}
A novel approach for performing the reverse time of flight (RTOF) analysis of the diffraction spectra through the use of time-delayed neural network (TDNN) is introduced. The new design applies a data acquisition system, a special interface card and software program installed in a PC computer, to perform the cross-correlation functions between signals received from the chopper-decoder and detector devices. This paper focuses on theoretical analysis of the neural network architecture to be embedded in the data acquisition system. The implementation of the suggested TDNN along with the experimental measurements will be deferred to a subsequent paper.
\end{abstract}

Index Terms-Reverse time of flight, data acquisition, time-delayed neural networks, cross-correlation.

\section{INTRODUCTION}

The neutron time-of-flight (TOF) diffractometers have been used widely for studying the properties of condensed matter. The conventional design TOF diffractometer does not provide a sufficient level of intensity and resolution at low power steady state reactors, using only on the order of 0.1 to $0.5 \%$ of the available neutrons [1]. Correlation TOF methods, using Fourier or pseudo-random beam modulation, have been developed for using the available neutron flux in a way more economic than the usual Fermi chopper system. In the pseudo-random method the beam intensity is modulated with a periodic binary pseudo-random signal (Fig.1A) and the time of flight spectrum is calculated as the cross-correlation of the modulation signal and the detector output [2]. The Fourier diffractometer involves modulation of the neutron beam by a fast Fourier chopper [3-7] (Fig.1B) which is a rotating disk (rotor) with a pattern of alternating neutron absorbing and neutron transparent slits; and a fixed system of

A)

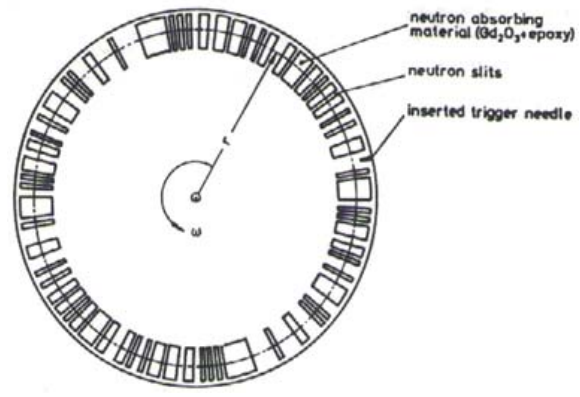

Fig.1: A) Pseudo-random chopper identical slits (stator). The method consists of measuring the Fourier transform of the TOF spectrum at several discrete frequencies and forming the spectrum itself by Fourier synthesis from the measured frequency components. The Fourier approach allows a duty factor up to $50 \%$ of the available neutrons. The Fourier method has been improved by the reverse time-of-flight (RTOF) concept [8-10], which is based on triggering of the TOF analyzer by the detected neutrons instead of the rotor's position. It should be emphasized that in contrast to the pseudo random and Fourier methods the RTOF is an on-line method. The basic idea of the RTOF method is an on-line check, for each detected neutron, of whether the registration probability is high or low. The check is realized by reverse analysis of neutron source and Fourier chopper states at the time the neutron passed through the corresponding points of the flight path. By carrying out neutron detection with the chopper speed continuously changing according to a particular law (frequency window), and recording only those neutrons with high probability of registration in the analyzer's memory, one can get the TOF distribution of elastically scattered neutrons, i.e., the conventional TOF diffraction pattern.

The layout of the RTOF diffractometer is schematically given in Fig.(2); where The incoming neutron beam is modulated by the chopper according to some function $x(t)$, whose values are always in the range $0 \leq x(t) \leq 1$. This function must naturally be a periodic function its frequency is varied in an appropriate manner during the experiment and is independent of neutron velocity. The transmitted neutrons are scattered by the sample into the detector, the output is used to start the multichannel analyzer (see Fig.2)

B)

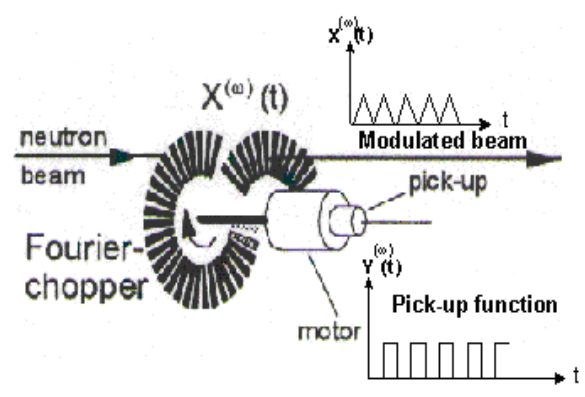

B) Fourier chopper 


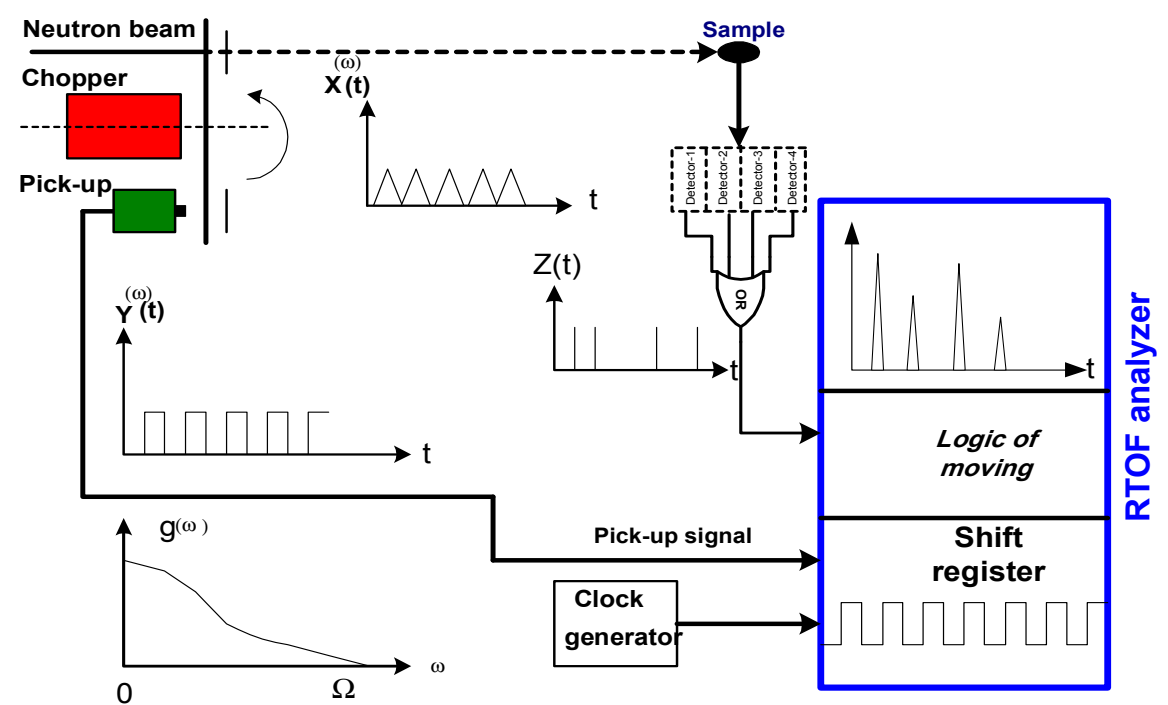

Fig. 2 : Schematic of the RTOF diffractometer operation principle

Applying the convolution theorem, it is seen that these start pulses form a nonhomogeneous Poisson process, with the intensity [4]:

$$
z(t)=\int_{0}^{T_{S}} S_{0}(\tau) x(t-\tau) d \tau+b_{0}
$$

where, $S_{0}(\tau) d \tau$ represents the counting rate of neutrons of flight times between the chopper and the detector are in the interval $(\tau, \tau+d \tau)$, and $\mathrm{b}_{0}$ is a constant background. The quantity to be measured is the TOF distribution $S_{0}(\tau)$, which includes the incoming neutron intensity and may be assumed to be non-zero only in a finite range $0<\tau<T_{s}$. The phase of the chopper is observed by a pick-up device, its output signal must accurately follow the modulation function $x(t)$ applied to the neutron beam up to the highest frequencies used. This signal $(y(t))$ is then delayed in the shift register and fed through the sampling gate into the analyzer. The shifting and sampling pulses as well as the channel advance signal for the analyzer are taken from the same clock-pulse generator. Thus, if the clock period is $\Delta$, and the shift register has $\mathrm{L}_{\mathrm{d}}$ stages, the analyzer input is essentially $y\left(t-T_{d}\right)$, where the delay is $T_{d}=\left(L_{d}-1\right) \Delta$. We note that the signal $y(t)$ would be a square wave with only two possible values (0 and 1) indicating whether $x(t)$ is below or above preset value. To explain the operation of the system as a whole we consider a measurement started at time $\mathrm{t}=0$, by dividing the time axis into intervals $(n-1) \Delta<t<n \Delta$, where $n=1,2, \ldots, L_{m}$, corresponding to a total measuring time equal to $T_{m}=L_{m} \Delta$. Assuming that the analyzer has $L_{a}$ channels of width $\Delta$ and we label them by $j=1, \ldots \ldots, L_{a}$. Now if a detector pulse triggers the measuring cycle of the analyzer during interval " $n$ ", the system will function in such a way that the contents of the $j^{\text {th }}$ analyzer channel are increased by: $y\left[n \Delta+(j-1) \Delta-T_{d}\right]$. In other words, if $\hat{Y}_{j}(n)$ denotes the contribution to be added into channel $\mathrm{j}$ due to the possible occurrence of a detector pulse in interval $\mathrm{n}$, it follows that we have for all $j$ :

$$
\begin{aligned}
& \widehat{Y}_{J}(n)= \\
& \left\{\begin{array}{c}
y\left[\left(n-L_{d}+j\right) \Delta\right], \text { if the analyzer is started during } n, \\
0, \text { otherwise }
\end{array}\right.
\end{aligned}
$$

The total spectrum that will be accumulated into the analyzer memory during the measurement time $T_{m}$ is then given by the channel contents:

$$
\hat{Y}_{j}=\sum_{n=1}^{L_{m}} \widehat{Y}_{j}(n), \quad j=1,2, \ldots \ldots, L_{a}
$$

The quantities $\hat{Y}_{j}(n)$ are random variables, but they are not independent with respect to $\mathrm{n}$, since, even at moderate neutron intensities, some detector pulses would occur during the recording cycles of the analyzer, thus giving no contribution to the spectrum $\hat{Y}_{j}$. It is therefore assumed here that the mean counting rate at the detector is so low that practically every pulse is likely to start the analyzer, i.e. we require that $\bar{Z} \ll 1 / T_{a}$, where $T_{a}=L_{a} \Delta$ is the dead time of the analyzer. We may treat the measured spectrum values $\hat{Y}_{j}$ as sums of $L_{m}$ independent, binomially distributed random variables, having the two possible outcomes defined in Eq.2. In particular, the probability for $\hat{Y}_{j}(n)=y\left[\left(n-L_{d}+J\right) \Delta\right]$ Will now be equal to the probability that a detector pulse occurs in interval $n$. Since $\Delta$ is normally very small, this probability can be approximated as:

$$
\pi_{\mathrm{n}}=\int_{(n-1) \Delta}^{n \Delta} z(t) d t \ll 1
$$

so that $1-\pi_{\mathrm{n}}$ is the probability for $\hat{Y}_{j}(n)=0$. So, the expectation value of $\hat{Y}_{j}$, which equals to the contents of channel $\mathrm{j}$ after measurement time $T_{m}=N \Delta$, is

$$
Y_{j}=E\left\{\hat{Y}_{j}\right\}=E\left\{\hat{\pi}_{n} \hat{y}_{j}\right\}=\sum_{n=0}^{N-1} \int_{n \Delta}^{(n+1) \Delta} z(t) y(t-j \Delta) d t
$$

The sum of the integrals over clock periods can be merged into one integral over the measurement time $T_{m}$ : 


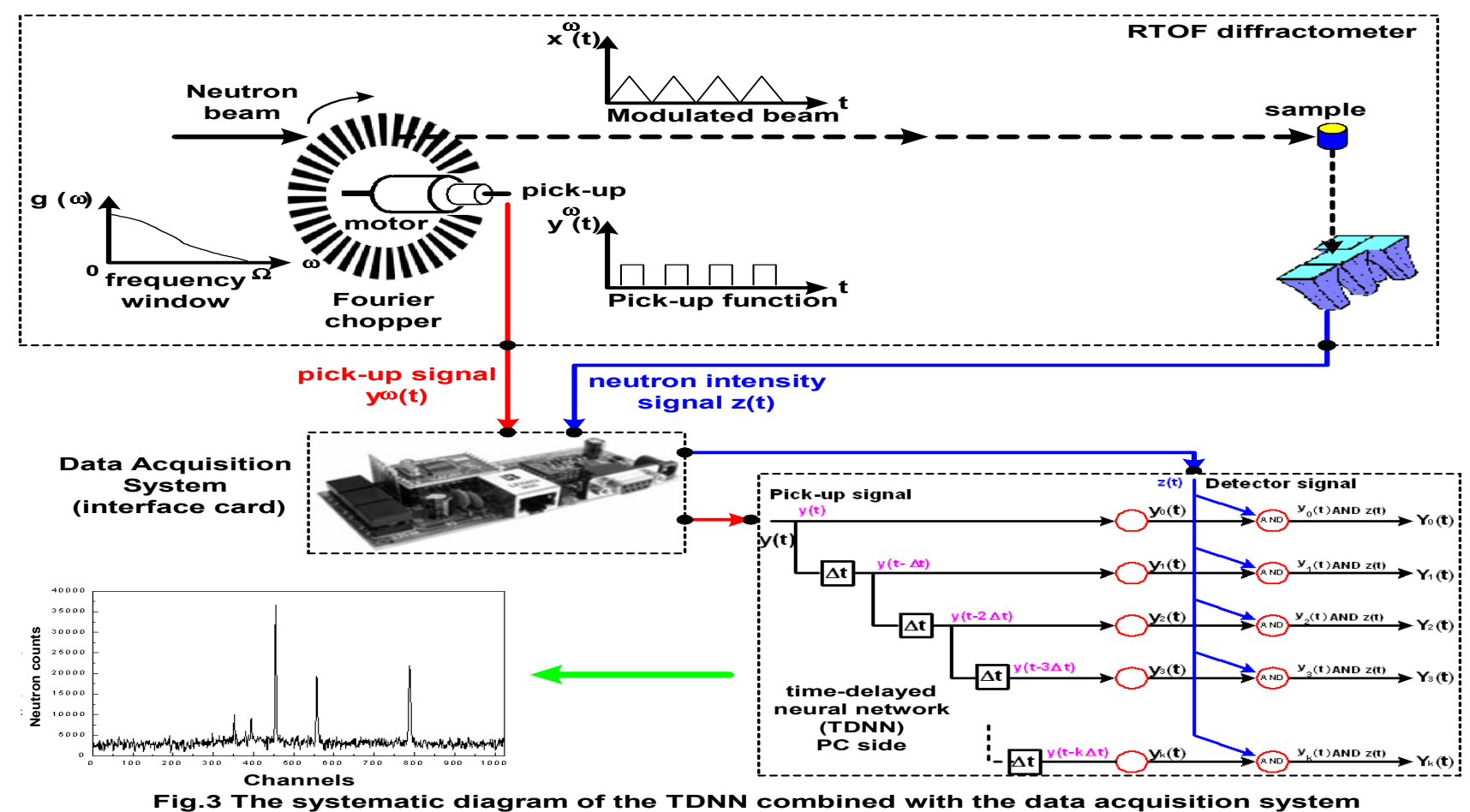

$$
Y_{j}=\int_{0}^{T_{m}} z(t) y\left(t-\tau_{j}\right) d t
$$

So, in the RTOF analyzer, the content of channel $j$ is the cross-correlation function of the detector intensity $z(t)$ and the clocked pickup signal.

There were many attempts to develop the current RTOF analyzer by replacing it with PC-based data acquisition systems $[11,12]$.

This paper discusses the ability to perform the cross-correlation functions between the acquired pick-up and the neutron intensity signals coming out from the interface card of the PC-based RTOF data acquisition system by utilizing a time-delayed neural network (TDNN).

Neural networks are networks of interconnecting neurons that compute specific functions when given a set of input signals [13]. When specific appropriate learning-rules are used in these networks, they can compute adaptive complex functions transforming the input into output that may not be solved by traditional analytical techniques, such as self-learning. The ability to perform these unique functions by these neural networks lies in (1) the neural network architecture (nonlinear multi-layered network), (2) the learning-rules, and (3) adaptive connection weights. These neural networks have been used in recent years to perform parallelizable computing functions that are capable of learning using the adaptive learning-rules to update their connection-weights. The significance of the computation performed by these networks depends very much on the network architecture and learning-rules [14,15].

\section{PROPOSED APPROACH}

The present work introduces new time-delayed neural network (TDNN) architecture to process time-varying signals The new approach applies a previously designed and tested data acquisition system, a special interface card and software program installed in a PC computer. The interface card receives the pick-up and the neutron intensity signals from the chopper and detector respectively. The function of the interface card then is to adapt the data collected from these input signals in order to be ready in the binary form at the time the program makes access to the interface card. The data acquisition system together with the neural network will act as substitute for the RTOF analyzer of the Fourier diffractometer (Fig.3). This neural network takes the adapted pick-up and neutron intensity signals as its inputs for

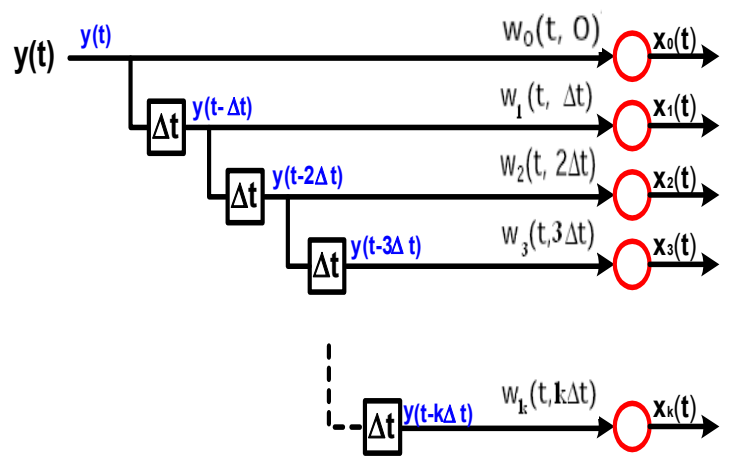

Fig.4 Architecture of the time-delayed neural network

processing in order to compute the cross-correlation function automatically. This time-delayed network is similar but different from the hybrid network introduced earlier by Tam $[16,17]$. The pick-up signal, as input, is delayed successively by a time-delay element in each input stage of the network (see Fig. 4). Thus, the time-delay produces the modified Hebbian learning-rule such that the connection-weight will change only if the time-delayed input and current output are activated rather than if the current input and current output are activated simultaneously. In other words, the output is associated with the previous input rather than the current input. Let $y(t)$ denotes the input pick-up signal at time $t$ and 
$y(t-\tau)$ denotes the signal delayed by the lag time $\tau$ and according to the time-delayed Hebbian learning-rule:

$$
\Delta w_{k}(t, k \Delta t)=y(t-k \Delta t) x_{k}(t)
$$

where $k$ is an integer constant and $\Delta w_{k}(t, k \Delta t)$ is the change in the $k^{\text {th }}$ connection-weight between the time-delayed input, $y(t-k \Delta t)$, and the $k^{\text {th }}$ output, $x_{k}$

The discrete lag-times $(\tau=k \Delta t)$ in integral, $k$, multiples of $\Delta t$ to delay the input signal by multiple delay-tap lines is used in the previous equation. A single time-series signal is used as the input to the network. This time-delayed input is cascaded into multiple branches as inputs to successive neurons to provide the inputs for the modified Hebbian learning-rule (Eq. 7) to update the corresponding connection-weights. The network would produce as many outputs as there are discrete time-delays. The $k$-th output of the network in Fig. 4 is given by:

$$
x_{k}(t)=w_{k}(t, k \Delta t) y(t-k \Delta t)
$$

Alternatively, each of the delay-tap lines in Fig.(4) can be considered as feeding into a pseudo-neuron as the first (pseudo) layer of the network in Fig.(5).

This first layer can be considered as a pseudo-layer for the

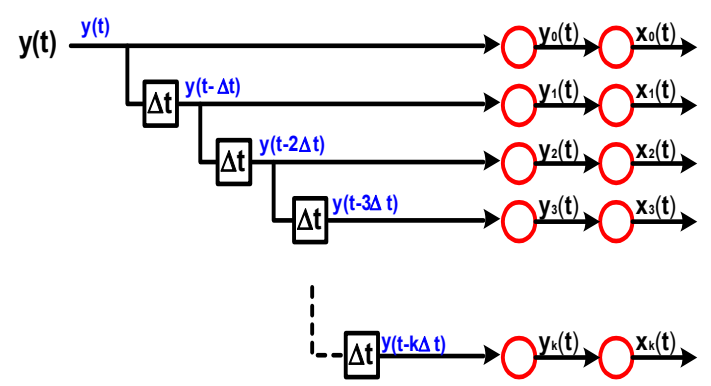

Fig.5 The time-delayed neural network with pseudo-layer

network because it does not perform extra computation, except for conceptualization of the equivalent neural network architecture. The output of the $\mathrm{k}^{\text {th }}$ time-delayed pseudo-input neuron can be expressed in terms of the initial input signal by:

$$
Y_{k}(t)=y(t-k \Delta t)
$$

The layer of time-delayed inputs is now represented as a parallel layer rather than a cascaded sequential input layer. Accordingly, the single sequential time-series input is transformed into parallel inputs by the delay lines allowing for simultaneous parallel processing rather than sequential processing. The neutron intensity signal can now be applied to the neurons of the input layer giving rise the neural network shown in Fig.6. The delayed pick-up $y(t-k \Delta t)$ signal will ANDed with the neutron intensity signal $z(t)$ point-by-point during the measurement time $T_{m} . \quad Y_{k}(t)$ after iterating $\mathrm{n}$ discrete time steps becomes:

$$
\mathrm{Y}_{\mathrm{k}}(j \Delta t)=\sum_{j=0}^{n} \mathrm{y}(\mathrm{j} \Delta \mathrm{t}-\mathrm{k} \Delta \mathrm{t}) \mathrm{z}(\mathrm{j} \Delta \mathrm{t})
$$

The delay lines of the suggested TDNN can be implemented through a circular linked-list data structure with $\mathrm{N}$ elements. The first and last elements of the linked-list are shifted right with one step every time-channel clock event allowing the insertion of the new coming pick-up pulse in the last element. At the same time the detector signal is checked and if it is logical " 1 ", the contents of the linked-list will be added to the corresponding output cells of the neural network.

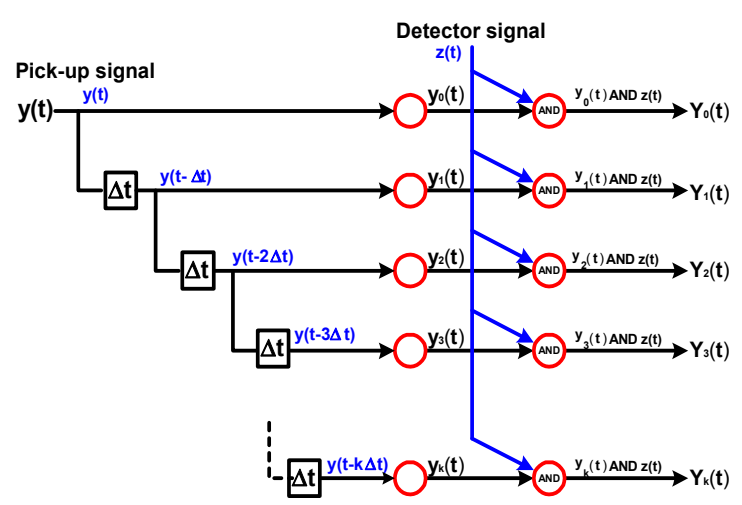

Fig.6 The architecture of the neural network that computes $y(t) \oplus z(t)$

\section{CONCLUSION}

The neural network architecture introduced in this paper satisfies the main objective of creating a neural network that correlates the chopper-decoder and detector signals of the RTOF diffractometer. The complete implementation of this design along with experimental measurements will be deferred to a subsequent paper.

\section{REFERENCES}

[1] V.A. Kudryashev and U. Hartung; Nucl. Inst. \& Meth.; A311, 563 (1992).

[2] K. Skold; Nucl. Inst. \& Meth.; 63, 114 (1968).

[3] A.Virjo; Nucl. Inst. \& Meth.; 73, 189 (1969).

[4] H. Poyry; Nucl. Inst. \& Meth.; 156, 499 (1978).

[5] P. Hiismaki, J. Junttila and A. Piirto; Nucl. Inst. \& Meth.; 126, 435 (1975).

[6] H. Poyry, P. Hiismaki and A. Virjo, Nucl. Inst. \& Meth.; 126, 421 (1975).

[7] A.Titta and A. Reverse, "Time-of-Flight Diffractometer using a Fourier Chopper", Technical Research Centre of Finland (VTT) Report 27, Espoo (1980).

[8] P. Hiismaki, P. Poyry and A. Tiitta; J. Appl. Cryst.; 21, 349 (1988).

[9] J. Schroder, et al.; J. Neutron Research; 2(4), 129 (1994).

[10] V.L. Aksenov, et. al.; J. Neutron Research; 5, 181 (1997).

[11] R.M.A. Maayouf and M.I. Khalil; Nucl. Inst. \& Meth.; 484, 459 (2002).

[12] M.I. Khalil; Meas. Sci. Technol.; 16, 1 (2005).

[13] E. Oja; Neural Networks; 5, 927 (1992).

[14] E. Oja; J. Math. Biol.; 15, 267 (1982).

[15] Y. Chauvin; Proc. IJCNN, Washington, DC; 1, 373 (1989).

[16] D. C. Tam; Progress in Neural Networks; 2, 129 (1994).

[17] D. C. Tam; The Open Cybernetics and Systemics Journal; 1, 1 (2007).

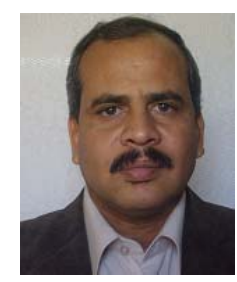

M. I. Khalil. received his B.Sc degree in Computer and Automatic Control Engineering from Ain Shams University, Cairo, Egypt, in 1983, M.Sc degree in Computer Engineering from Tanta University,Tanta, Egypt, in 2003 and Ph.D degree in Computer System Engineering from Benha University, Cairo, Egypt, in 2005. He is currently working as Assistant Professor in Department of Computers at the College of Science, Princess Noura Bent Abdulrahman University, Riyadh, KSA. He has 15 years of previous experience at the Reactor physics Department, Nuclear Research Center, Cairo, Egypt in the field of Data Acquisition and Interface Design. His area of interest includes image processing and Digital Signal processing. 